\title{
Mitofusin-2 Triggers Cervical Carcinoma Cell Hela Apoptosis via Mitochondrial Pathway in Mouse Model
}

\author{
Weiqiong Wang ${ }^{\mathrm{a}} \quad$ Xiaowen Liu ${ }^{\mathrm{a}, \mathrm{b}} \quad$ Xiaomei Guo ${ }^{\mathrm{a}} \quad$ Huanhuan Quan ${ }^{\mathrm{a}}$ \\ aDepartment of Cardiology, Tongji Hospital, Tongji Medical College, Huazhong University of Science \\ and Technology, Wuhan, ${ }^{b}$ Central Experiment Laboratory, the First College of Clinical Medical Sciences, \\ China Three Gorges University, Yichang, China
}

\section{Key Words}

Mfn2 $\cdot$ HeLa $•$ Apoptosis $•$ Bax $・$ Bcl-2 $\cdot$ Mitochondrial pathway

\begin{abstract}
Background/Aims: Cervical carcinoma continues to be one of the most dangerous cancer types, and more effective therapies are urgently needed for cervical carcinoma treatment. Mitochondria-associated Mitofusin 2 has influence on the progression of many cancers. In the current study, we aimed to focus on the cell apoptotic effects of Mfn2 on cervical carcinoma HeLa cells in vitro and to try to explore its underlying mechanisms. Moreover, we investigated the anticancer potential of Mfn2 in a cervical carcinoma mouse model. Methods: AdenovirusMfn2 (Adv-Mfn2) was used to deliver mfn2 into HeLa cells and tumour tissues in a nude mouse model. CCK-8, TUNEL assay, Western blot and immunohistochemical staining were performed to detect the effects of Mfn2. The mRNA level of Mfn2 was determined by quantitative realtime PCR (qRT-PCR) analysis. The effect of Mfn2 on cell apoptosis was investigated by flow cytometry. Flow cytometry was used to assess the change of the mitochondrial membrane potential of the cells treated with JC-1 assay. Mfn2, Bax, Bcl-2, cytochrome c, cleaved caspase-3, and cleaved caspase-9 protein levels were analysed by Western blot. Results: Data from CCK8 and flow cytometry showed that Mfn2 could inhibit proliferation and induce apoptosis in a dose- and time-dependent manner in HeLa cells. JC-1 test results revealed that the membrane potential of the mitochondrial decreased in a dose-dependent manner in HeLa cells after Adv-Mfn2 treatment. The data from Western blot confirmed that higher cytosolic amounts of cytochrome $\mathrm{c}$ with increasing doses of Adv-Mfn2 signified the onset of the intrinsic apoptotic pathway. Levels of cleaved caspase-3 and cleaved caspase-9 increased in HeLa cells with AdvMfn2 treatment. We also found significant increases in the Bax level and a decreased Bcl-2 level with Adv-Mfn2 treatment. We further confirmed that Mfn2 could significantly inhibit the growth of the cervical tumour in the xenografted cervical carcinoma mouse model. After a 9-day-treatment, the tumours of the Adv-mfn2 group were inhibited and induced into apoptosis. The results demonstrated that the overexpression of Mfn2 could not only increase the levels of Bax and Bid in cervical tumour cells but also decrease the phosphorylation of Bad
\end{abstract}

$X$. Liu and X. Guo contributed equally to this work. 
and the expression of $\mathrm{BCl}-2$. Conclusion: These studies suggested that the overexpression of Mfn2 could trigger cervical tumour apoptosis in vitro and in vivo, which was related to the mitochondrial pathway, and may provide a new treatment target for cervical carcinoma.

(C) 2018 The Author(s)

Published by S. Karger AG, Basel

\section{Introduction}

Based on the GLOBOCAN estimates, approximately 14.1 million new cancer cases and 8.2 million deaths occurred in 2012 worldwide. Over the years, the burden has shifted to less developed countries, which currently account for approximately $57 \%$ of the cases and $65 \%$ of the cancer deaths worldwide [1]. Targeting single molecular abnormalities or cancer pathways, which could modestly affect survival in some cancers, has achieved good clinical response [2]. Capabilities acquired as cancer hallmarks, including resisting cell death and so on, have stood the test of time as being integral components of most forms of cancer [3, 4]. These hallmarks contain many treatment targets, like the "apoptotic trigger" that conveys signals between the regulators and effectors, which is controlled by counterbalancing proand anti-apoptotic members of the Bcl-2 family of regulatory proteins [5].

The mitochondria controls many vital cellular parameters, such as the balance between mitochondrial fission and fusion, which affects the morphology of the mitochondria and also helps the mitochondria change the cytosolic localization, like accumulating where high amounts of ATP required or where $\mathrm{Ca}^{2+}$ signalling needs to be regulated $[6,7]$. Fusion of the mitochondria can attenuate stress by mixing the contents of partially damaged mitochondria as a form of complementation, and fission could not only lead to new mitochondria but also contribute to quality control by removing the damaged mitochondria [8-10].

The mitofusins (Mfn1 and Mfn2) associate with mitochondria and alter the mitochondrial morphology when expressed by transient transfection in tissue culture cells, which are homologs of the Drosophila protein, fuzzy onion (Fzo), and have been shown to affect the mitochondrial morphology when overexpressed yet play a reciprocal role in shaping the mitochondrial morphology in mammals $[6,11]$.

Mitofusin-2 (Mfn2) participates in mitochondrial fusion and contributes to the maintenance of the mitochondrial network [12-15]. The domain structure of the Mfn2 protein contains a p21 $1^{\text {ras }}$ signature motif, a GTP binding site motif, a possible PKA/PKG phosphorylation site, and a hydrophobic transmembrane domain [16]. An internal region, including a predicted bipartite transmembrane domain, is sufficient to target Mfn2 to the mitochondria and requires hydrophobic residues within the transmembrane domain [11].

Mfn2 is involved in the pathogenesis of hyper-proliferative diseases and in cancers as well, which makes Mfn2 a powerful cell proliferation suppressor [13, 16-18]. Studies on vascular hyper-proliferative disorders have proven that Mfn2 functions as a cell proliferation suppressor in serum-evoked vascular smooth muscle cells (VSMCs) in culture and in blocked balloon injury-induced neointimal VSMCs, whereas the over-expression of Mfn2 could markedly suppress restenosis in rat carotid arteries [16].

Notably, previously unappreciated effects of Mfn2 in promoting apoptosis have immediately been noticed $[13,19]$. The Mfn2 gene is localized in the human chromosome $1 \mathrm{p} 36.22[6,16]$, whereas chromosome 1 aberrations are related to various human cancers, such as neuroblastoma, breast cancer and cervical cancer, which may suggest the existence of tumour suppressor genes in this region [20,21]. Recently, the pro-apoptotic and antiproliferative effects of Mfn2 in different types of cancers have been studied, including liver, breast and urinary bladder cancers [22-24]. Mfn2 is frequently lost in breast cancer, which has influence on breast cancer progression by a new mitochondria reprogramming pathway [17], and the PI3K-AKT-mTOR pathway plays an important role in the activation-induced downregulation of Mfn2 and the subsequent proliferation of resting human T cells [25]. In our early study, we found that the PTD4-apoptin fusion protein could induce HeLa cell apoptosis and upgrade Mfn2 expression [26]. However, the effects of Mfn2 in cervical cancer have rarely been investigated, and the underlying mechanism is worth researching. 


\section{Cellular Physiology Cell Physiol Biochem 2018;46:69-81

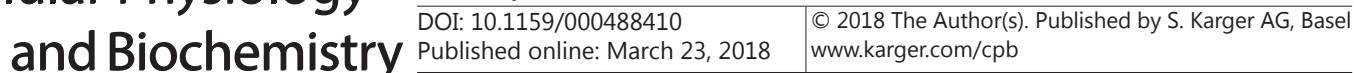

Wang et al.: Mfn2 Induces Hela Cells into Apoptosis

In the current study, we focused on the cell apoptotic effect of Mfn2 on the cervical carcinoma HeLa cells in vitro and tried to explore the underlying mechanisms. Moreover, we investigated the anticancer potential of Mfn2 in vivo in acervical carcinoma mouse model.

\section{Materials and Methods}

\section{Antibodies}

Antibodies used were as follows: Anti-Mfn2 (Abcam, USA) directed against the Mfn2 protein, p53 (Cell Signalling Technology, USA), cleaved caspase-3 (Cell Signalling Technology, USA), cleaved caspase-9 (Cell Signalling Technology, USA), Bcl-2 (Affinity Biosciences), Bid (Affinity Biosciences), Bax (Cell Signalling Technology, USA), p-Bad (Cell Signalling Technology, USA) and cytochrome c (Cell Signalling Technology, USA). GAPDH (Santa Cruz, USA) and $\beta$-actin (Tianjin Sungene Biotech, China) antibodies were used as internal loading controls.

\section{Construction of mfn2 expression adenoviral vector}

Rno-mfn2 precursor DNA (Homo sapiens (human), Gene ID: 9927) was synthesized by Genechem (Shanghai, China). The adenovirus expressing mfn2 (Adv-Mfn2), or controlling the control adenovirus expression (Adv-control) was generated using the AdMax system (Microbix Biosystems, Canada) according to the manufacturer's protocols. These resulting adenoviruses were further packaged and amplified in HEK293 cells and purified using CsCl banding. Viral titre was routinely concentrated to nearly $1 \times 10^{9} \mathrm{PFU} /$ $\mathrm{ml}$ as determined by a plaque assay.

\section{Cell culture}

The human cervical carcinoma cell line, HeLa, was purchased from the Chinese Culture Tissue Collection Centre and cultured in RPMI 1640 (Thermo Fisher Scientific, Beijing, China) supplemented with $10 \%$ foetal bovineserum (FBS, Gibco BRL Life technologies, USA) under a humidified $5 \% \mathrm{CO}_{2}$ atmosphere at $37^{\circ} \mathrm{C}$. Cells were checked routinely and trypsinized until they reached $80-90 \%$ confluency.

\section{CCK-8 test}

The cell viability was measured via a cell counting kit-8 (CCK-8 kit, Dojindo Molecular Technologies Inc., Shanghai, China) according to the manufacturer's instructions. Approximately $3 \times 10^{3}$ cells were seeded in $100 \mu \mathrm{l}$ per well RPMI 1640 or DMEM in a 96-well plate. The panel of HeLa cells or C33A cells was treated with various concentrations $(0,30,60,90,120,150 \mathrm{pfu} / \mathrm{ml})$ of Adv-Mfn2 or Adv-control, and the cells were cultured for different lengths of time ( $48 \mathrm{~h}$ and $60 \mathrm{~h}$ ). Subsequently, $10 \mu \mathrm{l}$ of the CCK-8 solution was added to each well and incubated at $37^{\circ} \mathrm{C}$ for another $2 \mathrm{~h}$. The absorbance at $450 \mathrm{~nm}$ was measured on a spectrophotometric plate reader. Each group was repeated in three different wells.

\section{Cell apoptosis analysis by flow cytometry}

HeLa cells were seeded in 6-well plates for $24 \mathrm{~h}$ before being exposed to Adv-mfn2. After HeLa cells were treated with 90 or 120pfu/ml of Adv-Mfn2 for $48 \mathrm{~h}$ and $60 \mathrm{~h}$, the cells were harvested, washed with pre-chilled PBS twice and prepared in a single-cell suspension. Apoptosis was measured by a flow cytometer (BD Biosciences, USA) with FITC-conjugated Annexin V and propidium iodide (PI) double staining (Nanjing Biobox Biotech. China).

\section{Mitochondrial membrane potential analysis by flow cytometry}

HeLa cells were seeded in a 6-well tissue culture plate. Adv-control and Adv-mfn2 were added into the medium after $24 \mathrm{~h}$. After the HeLa cells were treated for $60 \mathrm{~h}$ with 90 or $120 \mathrm{pfu} / \mathrm{ml}$ of Adv-Mfn2, the alteration of the mitochondrial membrane potential was measured using the Mitochondrial Membrane Potential Detection Kit (Beyotime Institute of Biotechnology, Nanjing, China). After incubation with JC-1 fluorescence dye for 30 mins, the cells were washed and resuspended with JC-1 Assay Buffer. Flow cytometry analysis was performed using a flow cytometer (BD Biosciences, USA).

\section{Xenografted cervical carcinoma mouse model}

BALB/c nude mice (4-5 weeks old) were obtained from the Hubei Provincial Centre for Disease Control and Prevention (HBCDC, China). We collected $6 \times 10^{5}$ human cervical carcinoma HeLa cells and injected them 


\section{Cellular Physiology Cell Physiol Biochem 2018;46:69-81 and Biochemistry Published online: March 23, 2018 \begin{tabular}{l|l} 
DOI: 10.1159/000488410 & $\begin{array}{l}\text { C } 2018 \text { The Author(s). Published by S. Karger AG, Basel } \\
\text { www.karger.com/cpb }\end{array}$
\end{tabular}}

Wang et al.: Mfn2 Induces Hela Cells into Apoptosis

subcutaneously in the right armpit of nude mice. When the tumours were visible, the mice were divided into 2 groups consisting of 8 tumour-bearing mice per group for a 9-day treatment with Adv-Mfn2 and Advcontrol, respectively. Adenovirus was injected into the tumour tissue. Every three days, a $100-\mu l$ solution of Adv-control $\left(5 \times 10^{8} \mathrm{pfu} / \mathrm{ml}\right)$ or Ad-Mfn $2\left(5 \times 10^{8} \mathrm{pfu} / \mathrm{ml}\right)$ was injected into the tumours per mouse, which were determined from our preliminary experiment. All animal studies were carried out in accordance with the "Guide for the Care and Use of Laboratory Animals" and approved by the Hubei Provincial Centre for Disease Control and Prevention (HBCDC, China).

Quantitative real-time PCR ( $q$ RT-PCR) analysis

Total RNA from the HeLa cells or tumour issues was extracted using TRIzol ${ }^{\circledR}$ Reagent (Invitrogen, USA). Reverse transcription and qRT-PCR were performed as described previously [27]. Amplification and detection of specific products was performed with ABI step one plus (PE Applied Biosystems). RevertAid Reverse Transcriptase (Thermo scientific, EP0442) and qPCR Master Mix (Fermentas, K0221) were used to measure the mfn2 levels. GAPDH was used as an internal control for mfn2 RNA. Data were analysed using the 2- $\Delta \Delta \mathrm{Ct}$ method. The following sequence-specific primers were used to amplify the gene products: mfn2, F:5'-ATCTGTGCCAGCAAGTTGACA-3', R:5'-AAGTGAATCCAGAGCCTCGAC-3', and GAPDH, F:5'GACAAAATGGTGAAGGTCGGT-3', R:5'-GAGGTCAATGAAGGGGTCG-3'.

\section{Western blotting}

Whole cell proteins were extracted with a RIPA lysis buffer in vitro experiment, while the tumour tissues in the xenograft experiment were homogenized with ice-cold lysis buffer for total protein extraction. Protein concentrations were determined using the BCA protein assay kit (Pierce, 23227) according to the manufacturer's instructions. The proteins were fractionated on a $12 \%$ and $15 \%$ SDS-polyacrylamide gel and electroblotted onto Immobilon-P PVDF transfer membranes (Millipore, IPVH08130), as recently reported [28]. The blots were incubated with Mfn-2, anti-p53, cleaved caspase-3, cleaved caspase-9, cyto c, Bax, Bcl2 , Bid and p-Bad antibodies. GAPDH and $\beta$-actin were used as loading controls. The positive signals were visualized by Odyssey ${ }^{\circledR}$ Two-Color Infrared Imaging System (Li-Cor, USA).

\section{Detection of xenografted tumour growth and TUNEL assay}

The tumour volume was analysed at $0,3,6$ and 9 days after starting the protein treatment, and the volume of the tumours was measured by the following formula: volume $=0.52 \times$ length $\times$ width $^{2}$ [28] . After a 9-day treatment, the tumour tissues were obtained by sacrificing the mice, and the tumours were dissected and fixed in 4\% paraformaldehyde. Paraffin sections were prepared for TUNEL assays (terminal deoxynucleotidyl transferase-mediated dUTP-biotin nick end labelling assay) by means of the In situ Cell Death Detection Kit, POD (Roche, 11684817910). Image-Pro Plus was used to quantify each picture.

\section{Immunohistochemistry}

After a 9-day treatment, the mice were sacrificed, and the tumours were fixed with 4\% paraformaldehyde. Paraffin sections were prepared for immunohistochemistry. The presence of cleaved caspase- 3 was analysed by means of corresponding antibodies that were recently reported in our paper [28]. Image-Pro Plus was used to quantify each picture.

\section{Statistical analysis}

Statistical analyses were carried out in GraphPad Prism. The Student's t-test was used to determine the statistical significance of the data. A $p$ value less than $0.05\left({ }^{*}\right)$ or less than $0.01\left({ }^{* *}\right)$ was considered to be significant. Data presented in the figures represent the mean \pm SD.

\section{Results}

Effects of Mfn2 on human cervical carcinoma HeLa cells in vitro

To examine the effects of Mfn2 on the viability of human cervical carcinoma HeLa cells, a panel of HeLa cells was treated with various concentrations (0 to $150 \mathrm{pfu} / \mathrm{ml}$ ) of Adv-Mfn2. Meanwhile, effects of Mfn2 on C33A cells, which were HPV negative cervical cancer cells, were 
investigated. As shown in Fig. 1A and 1B, the exposure of HeLa cells or C33A cells to AdvMfn2 resulted in a decrease in cell proliferation. Western blot analysis confirmed that Mfn2 expression increased in HeLa cells infected with Adv-Mfn2 compared with the Adv-control group and the blank control group (Fig. 1C). The data suggested that Mfn2 overexpression could inhibit HeLa cell proliferation in a concentration- and time-dependent manner.

\section{Mfn2 induced apoptosis of HeLa cells}

To examine the possible mechanism of Mfn2 inhibition of cell viability, apoptosis was investigated after Adv-Mfn2 treatment of HeLa cells. We speculated that Mfn2 could further lead to programmed cell death in HeLa cells due to the effects of Adv-Mfn2 on proliferation. To address this issue, we evaluated the apoptosis of HeLa cells treated with the indicated doses of Adv-Mfn2 for $48 \mathrm{~h}$ and $60 \mathrm{~h}$ using an Annexin V-FITC/PI staining assay. Phosphatidylserine

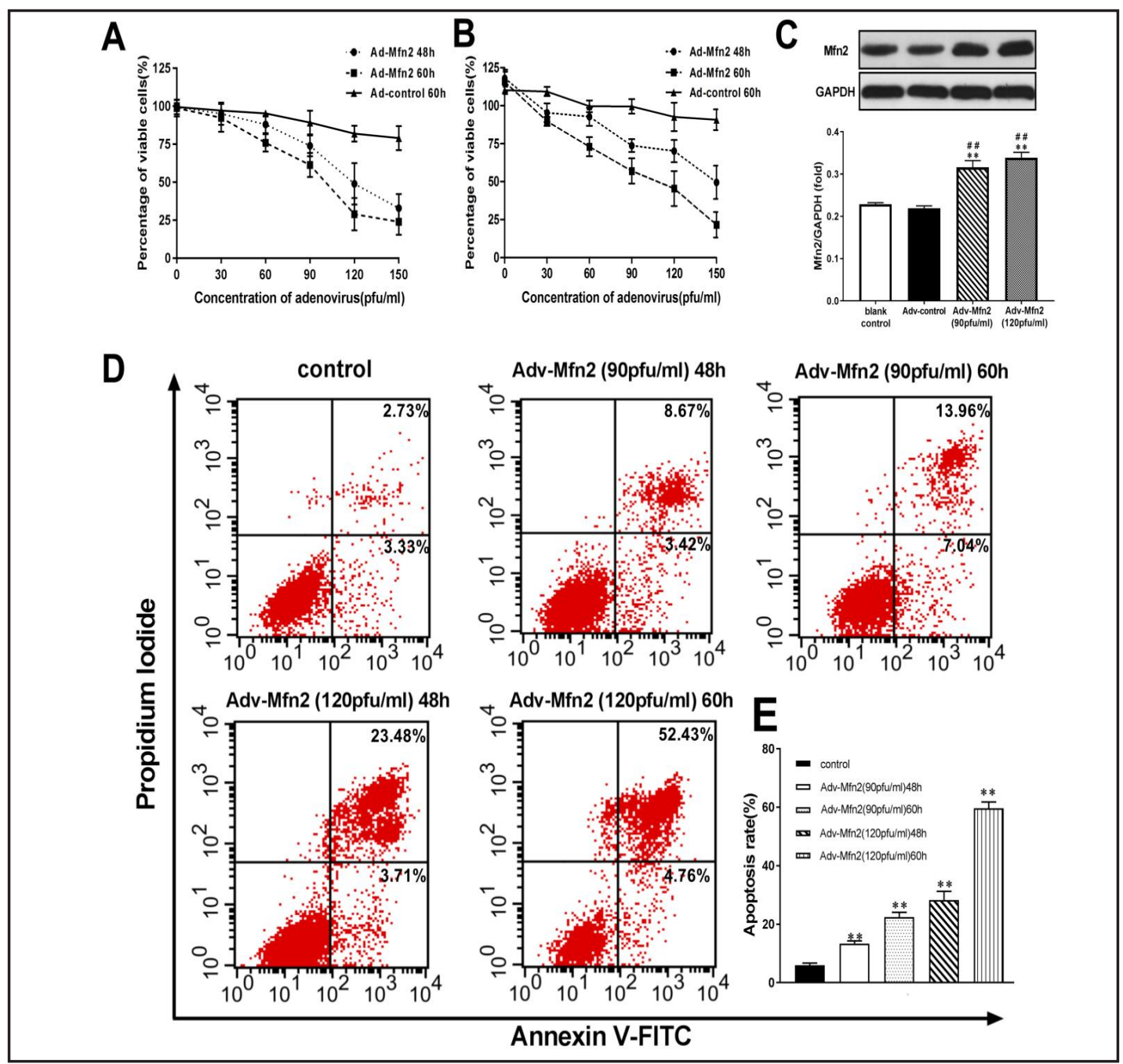

Fig. 1. The effects of Mfn2 on cell growth and apoptosis in HeLa cells. HeLa cells (A) and C33A cells (B) were exposed to different concentrations of Adv-Mfn2 (0,30, 60, 90, 120, $150 \mathrm{pfu} / \mathrm{ml})$ for $48 \mathrm{~h}$ and $60 \mathrm{~h}$ or Advcontrol for $60 \mathrm{~h}$ and then the cell viability was measured using a CCK-8 assay. (C) Cell lysate was extracted from HeLa cells treated with DMSO, Adv-control, or Adv-Mfn2 (90 or 120 pfu/ml) for Mfn2 expression by Western blot analysis. GAPDH was used as a reference. (D) HeLa cells were incubated with Ad-Mfn2 for 48 h or 60 h. Cells were stained with Annexin V-FITC/PI after incubation with two concentrations(90 or 120 $\mathrm{pfu} / \mathrm{ml}$ ) of Adv-Mfn2 were measured by flow cytometry. (E) The percentage of cell apoptosis after HeLa cells were treated with the indicated concentrations of Adv-Mfn2. All results are expressed as the mean \pm SD from three independent experiments ( ${ }^{* *} \mathrm{p}<0.01$ vs Adv-control group; \#\# $\mathrm{p}<0.01$ vs blank control group). 


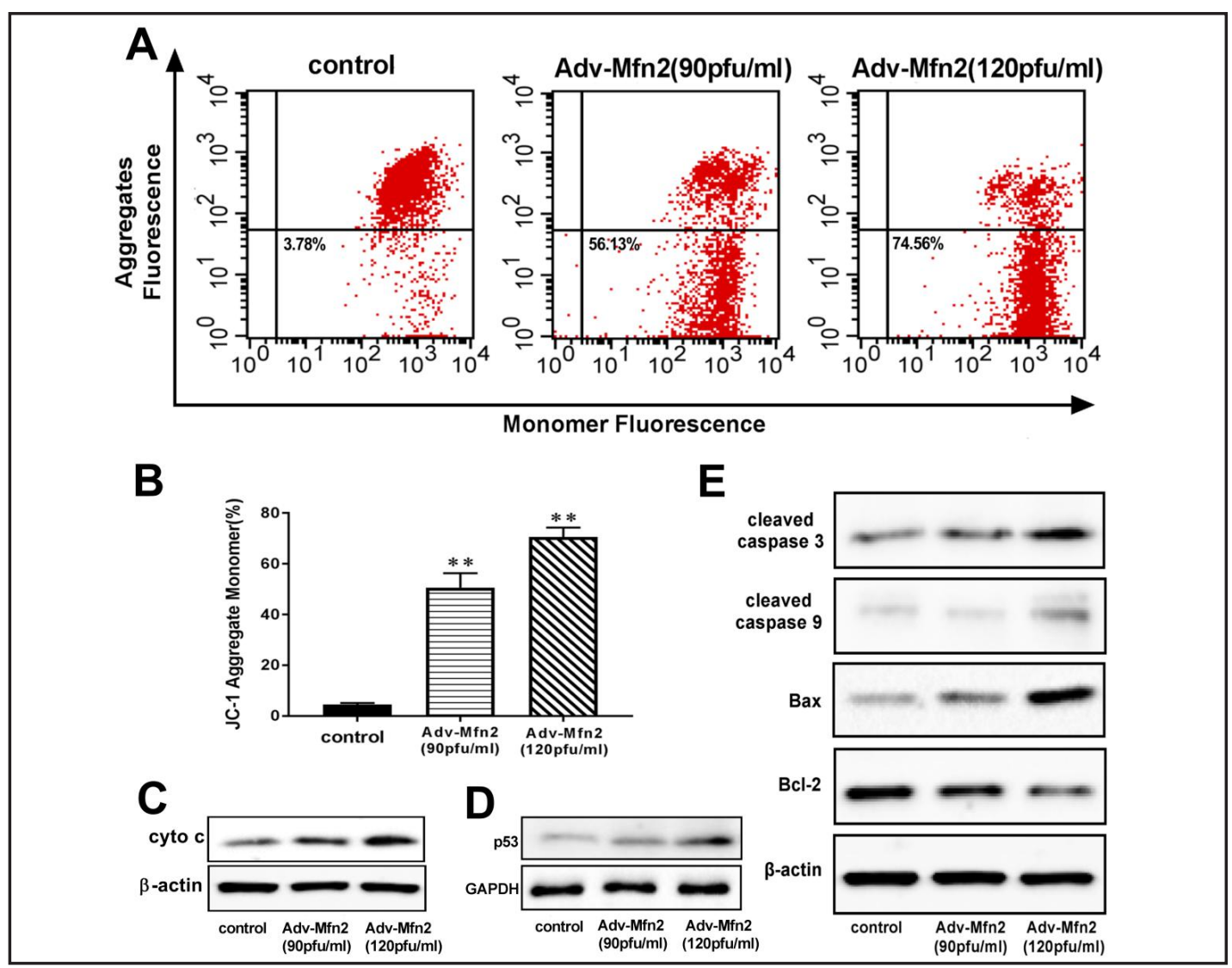

Fig. 2. Mitochondrial membrane potential decreased and the expressions of mitochondrial apoptosis-related proteins were regulated after being treated with Adv-Mfn2. (A) Mitochondria membrane potential decreased after HeLa cells were treated with Adv-Mfn2 for $60 \mathrm{~h}$ in a dose-dependent manner. (B) Dose-dependent changes in the mean JC-1 fluorescence after HeLa cells were treated with Adv-Mfn2 for $60 \mathrm{~h}$ (mean \pm SD from three independent experiments, ${ }^{* *} \mathrm{p}<0.01$ ). (C) Adv-Mfn2 increased the release of cytochrome c into the cytosol by Western blot. Beta-actin was used as reference. (D) The expression of p53 protein in the cervical tumour HeLa cells was detected by Western blot. GAPDH was used as a reference. (E) Expressions of the mitochondrial apoptosis-related proteins, such as cleaved caspase-3, cleaved caspase-9, Bax and Bcl-2 after HeLa cells were treated with Adv-Mfn2 for 60 h. Beta-actin was used as reference.

expressed on the cell surface, which is an event identified in apoptosis, can be detected using Annexin V as a probe. As shown in Fig. 1D and 1E, HeLa cells displayed apparent apoptosis after treatment with Adv-Mfn2 in a dose-dependent manner. Taken together, these data revealed that Mfn2 could induce apoptosis in HeLa cells.

\section{Mfn2 mediated mitochondrial membrane potential and cytochrome c release}

Apoptosis is often accompanied by a decrease in the mitochondrial membrane potential (MMP) due to the disruption of the mitochondrial integrity, which is an early event and standard evidence in mitochondrial pathway apoptosis.

The cyanine dye JC- 1 could form J-aggregates that are spectrally distinguishable from the dye monomers at high concentrations in the energized mitochondria of cells exposed to near-micromolar external concentrations of dye, which makes the JC-1 assay widely used for the measurement of MMP [29]. JC-1, as a monomer at low membrane potentials, and JC- 1 , as J-aggregates at high membrane potentials, could be measured by flow cytometry to determine the MMP. Therefore, we examined the changes of the MMP in HeLa cells after treatment with Adv-Mfn2 or Adv-control for $60 \mathrm{~h}$. Interestingly, we found that the mitochondrial membrane potential was clearly decreased in a dose-dependent manner (Fig. 2A and 2B). These data 


\section{Cellular Physiology Cell Physiol Biochem 2018;46:69-81

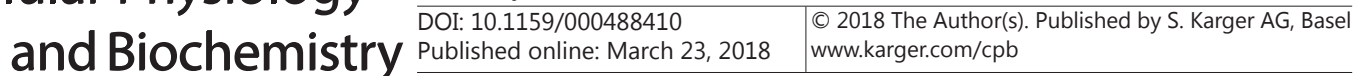 \\ Wang et al.: Mfn2 Induces Hela Cells into Apoptosis}

suggest that Mfn2 was able to affect the mitochondrial membrane potential of HeLa cells. Mitochondrial membrane depolarization is an early indicator of apoptosis, which causes the release of cytochrome $\mathrm{c}$ from the mitochondria into the cytosol and initiates a signalling cascade that executes cell death by apoptosis [30]. As evidenced by Western blot data (Fig. 2C), Adv-Mfn2 increased the release of cytochrome c from the mitochondria into cytosol.

\section{Mfn2 induced activation of caspase-3 and caspase-9 in HeLa cells}

Apoptosis is mediated by the activation of caspase cascades, which initiate and execute apoptosis. Earlier, it was reported that the activation of caspase- 9 was linked with the release of cytochrome c from the mitochondria into the cytosol and the induction of apoptotic signalling pathways [31]. Western blot results showed that the expression of p53 protein was obviously upregulated compared with the Adv-control group (Fig. 2D). To determine the role of caspase- 3 and caspase- 9 in the Mfn2-induced mitochondrial membrane depolarization in HeLa cells, we next examined the levels of activated caspase- 3 and caspase- 9 using Western blot. As shown in Fig. 2E, the higher activity of cleaved caspase-3 and cleaved caspase- 9 in HeLa cells was associated with higher Adv-Mfn2 concentration. The data suggested that the mitochondrial apoptosis pathway induced by Mfn2 in HeLa cells was related with the activation of caspase-3 and caspase-9.

Mfn2 regulated the expression of $B c l-2$ and Bax in the mitochondria apoptosis signalling pathway

The Bcl-2 family, which is important in the mitochondria apoptosis cascade, consists of pro-apoptotic and anti-apoptotic proteins. To further explore the molecular mechanism

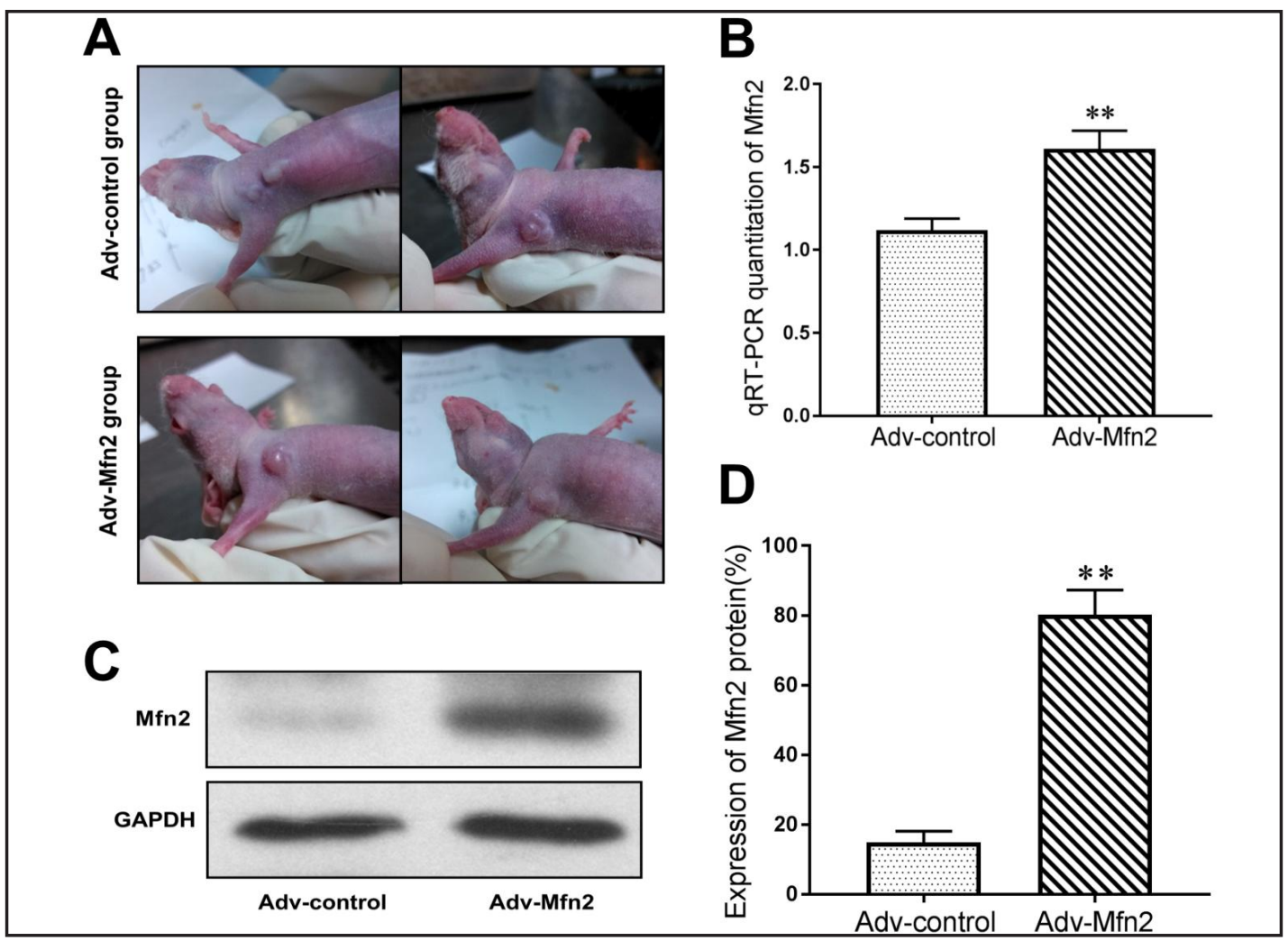

Fig. 3. Mfn2 is taken up into the tumours of the in vivo cervical carcinoma model. (A) The xenografted cervical carcinoma model in the right armpit of nude mice. (B) The expression of mfn2 RNAs in the tumours of the mouse model were detected by qRT-PCR quantitation. Data are represented as the mean \pm SD $\left({ }^{* *} p<0.01\right)$. (C) and (D) The expression of Mfn2 proteins in the tumours of the mouse model were detected by Western blot (C) and quantitation (D). Data are represented as the mean $\pm \operatorname{SD}\left({ }^{* *} \mathrm{p}<0.01\right)$. 
behind the mitochondria-dependent apoptosis induced by Mfn2, we examined the expression of Bcl-2 family proteins, including the pro-apoptotic protein Bax and anti-apoptotic protein Bcl-2. As seen in Fig. 2E, the overexpression of Mfn2 could upregulate the Bax protein level and downregulate the level of $\mathrm{Bcl}-2$ compared to the control group, which suggested that the decrease of the Bcl-2/Bax ratio was involved in apoptosis induced by Mfn2.

\section{Overexpressed Mfn2 in tumour tissues of the cervical carcinoma mouse model}

The human cervical carcinoma HeLa cells were injected subcutaneously on the right armpit of female BALB/c nude mouse. After 5 days, the tumour tissues were found on the right armpit of the mice (Fig. 3A), and the volumes were over $3 \times 3 \mathrm{~mm}^{2}$. The Adv-Mfn2 and Adv-control were injected into the tumours. The tumour tissues were obtained $72 \mathrm{~h}$ after injection with Adv-mfn2 or Adv-control, and the Mfn2 mRNAs were detected by qRT-PCR (Fig. 3B), while the proteins were examined by Western blot (Fig. 3C and 3D). We found that the RNAs and proteins of $\mathrm{mfn} 2$ were over expressed in tumour tissue after $72 \mathrm{~h}$ of treatment with Adv-Mfn2.

\section{Mfn2 inhibited the growth of cervical carcinoma in vivo}

To verify the effects of Mfn2 against cervical carcinoma in vivo, the therapeutic effects of Mfn2 on tumour tissues in the xenografted cervical carcinoma mouse model for a 9-daytreatment were assessed. HeLa cells were injected subcutaneously, and the animals were randomly divided into two groups of 8 tumour-bearing mice each. The bigger tumourbearing mice were treated with Adv-Mfn2, and the other group was treated with Advcontrol as described in the Materials and Methods section. At days 0, 3, 6 and 9 during the

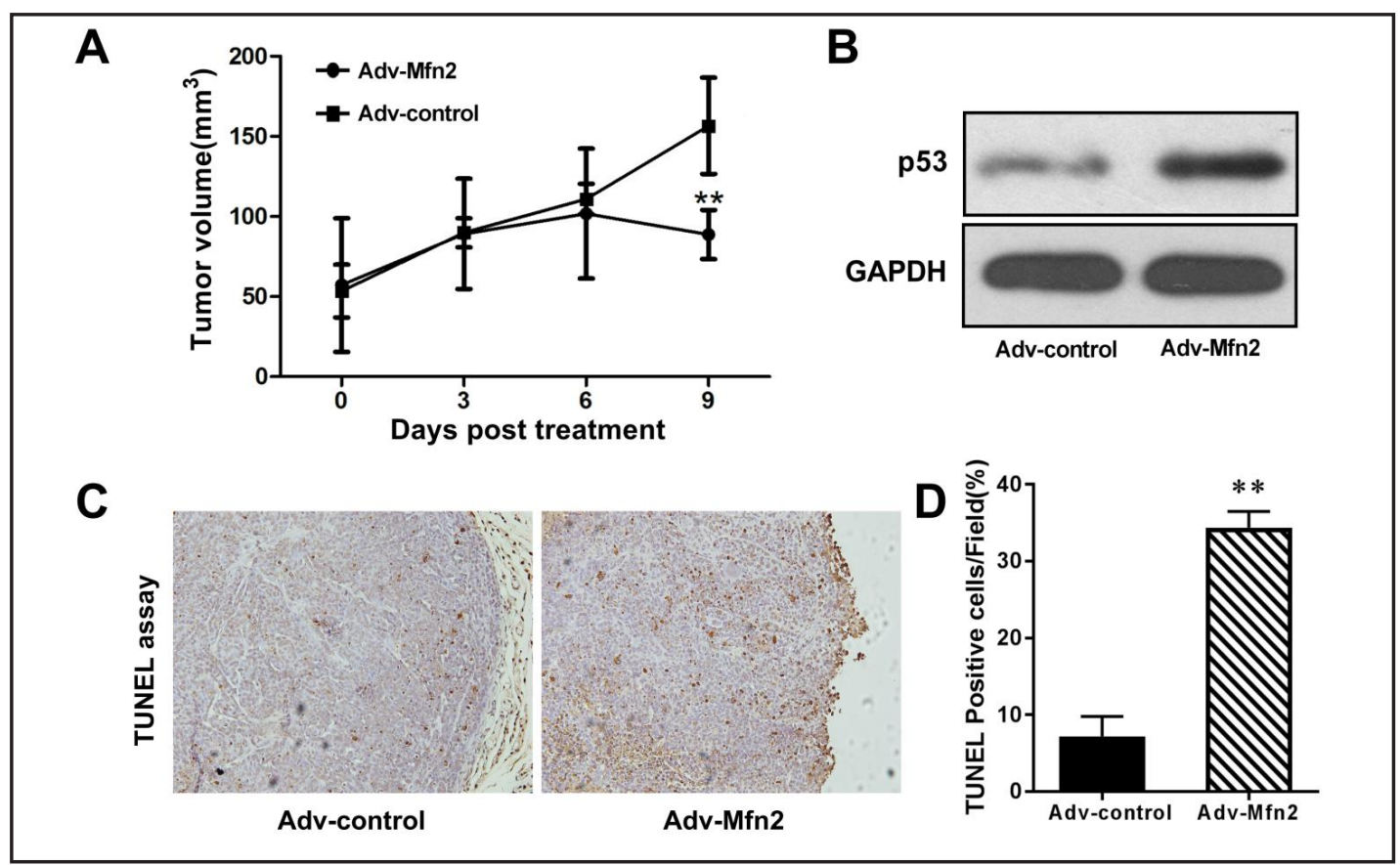

Fig. 4. Mfn2 inhibited tumour growth in an in vivo cervical carcinoma model. (A) Determination of the tumour volume in mice treated with Adv-mfn2 or Adv-control for 0, 3, 6 and 9 days. Data are represented as the mean $\pm \mathrm{SD}\left({ }^{* *} \mathrm{p}<0.01\right)$. (B) Western blot showed p53 protein levels in cervical tumour cells. The cervical tumour cells were prepared from total tumour tissue lysates and analysed by Western blot. GAPDH was used as reference. (C) TUNEL assay of the tumour sections from animals treated with either Adv-control or AdvMfn2. Cells that underwent apoptosis by Adv-mfn2 treatment were recognized by brown cell staining. The nuclei of the tumour cells appeared blue. Magnification: 200×. (D) Quantification of TUNEL positive cells of the tumour sections from animals treated with either Adv-control or Adv-Mfn2 (**p $<0.01$ vs Adv-control group). 


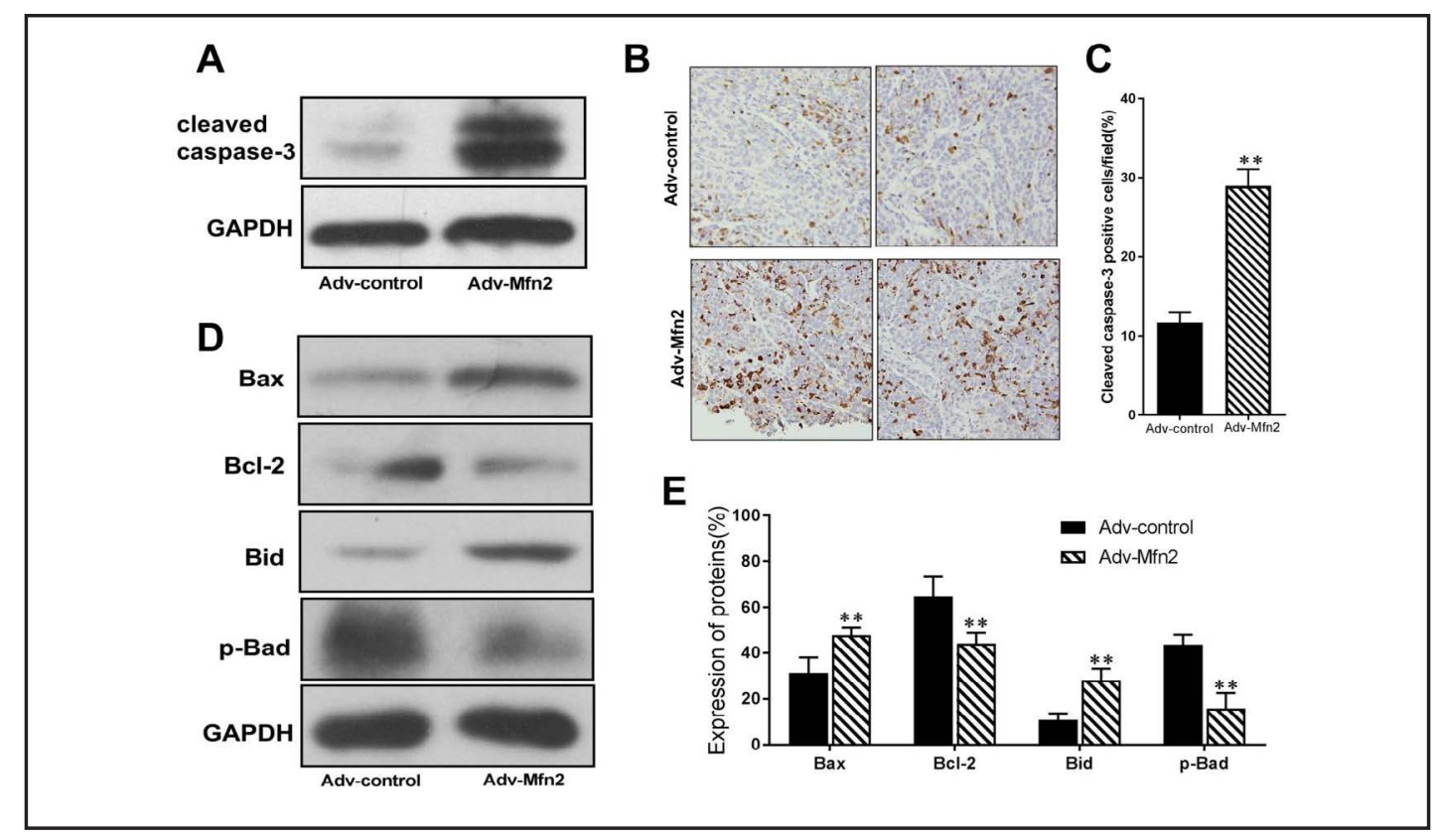

Fig. 5. Mfn2 triggered cervical tumour cell apoptosis via the mitochondrial pathway. (A) Western blot showed cleaved caspase-3 protein levels in the cervical tumours treated with Adv-mfn2 after 9-day-treatment. Total tumour tissue lysates were prepared and analysed by Western blot for cleaved caspase- 3 protein levels. GAPDH was used as reference. (B) The picture of the immunohistochemical staining represented the expression of cleaved caspase-3 protein levels between the Adv-Mfn2 group and Adv-control group as detected by anti-cleaved caspase-3 staining. Magnification: 400×. (C) Quantification of the expression of cleaved caspase-3 protein levels between the Adv-Mfn2 group and Adv-control group ( ${ }^{* *} \mathrm{p}<0.01$ vs Advcontrol group). Western blot (D) and quantitation (E) showed Bax, Bcl2, Bid and p-Bad protein levels in the cervicaltumour cells. The cervicaltumour cells were prepared from total tumour tissue lysates, and analysed by Western blot. GAPDH was used as reference.

treatment, the volume of the tumours was analysed (Fig. 4A). After treatment, the volume of the xenografted cervical carcinomas treated with Adv-mfn2 declined gradually, whereas the volume of the tumours treated with Adv-control increased. At day 9, the tumour tissues were obtained, and the expression of the p53 protein was obviously upregulated compared with the Adv-control group analysed by Western blot (Fig. 4B).

After the treatment, the mice were sacrificed, and the tumours were analysed by means of a TUNEL assay (Fig. 4C, 4D). The Adv-Mfn2 group underwent massive apoptosis, as indicated by the observed brown colouring in the analysed histological sections. In contrast, the tumours treated with Adv-control had no significant signs.

\section{Mfn2 induced Hela tumour tissues to apoptosis in mouse model}

To confirm the apoptosis induction in HeLa tumour tissues by Adv-Mfn2, the active executioner caspase- 3 was analysed by Western blot and immunohistochemical staining. As shown in Fig. 5A, Western blot analysis showed that caspase-3 was activated in tumours of the Adv-Mfn2 group compared with the Adv-control group after 9 days of treatment. Subsequently, sections of all the treated tumours were prepared for the detection of active executioner caspase- 3 by staining the tumour sections with anti-cleaved caspase- 3 antibodies directed against the cleaved caspase- 3 present in the cervical tumour tissues. Fig. $5 \mathrm{~B}$ and $5 \mathrm{C}$ showed that the cleavage caspase- 3 proteins appeared in large parts of the Adv-Mfn2-treated tumours compared with the control group. These data demonstrated that Mfn2 could reduce the size of xenografted cervical carcinoma in vivo and induced apoptosis in the cervical tumour.

\section{KARGER}


Mfn2 induced cervical carcinoma to apoptosis via the mitochondrial pathway

The Bcl-2 family, which is known to play a relevant role in the development of cervical carcinoma, comprises two groups of proteins with distinct functional roles in the intrinsic pathway of apoptosis in that they harbour anti-apoptotic or pro-apoptotic activity [29]. The Bcl-2 protein is an anti-apoptotic member that is discovered first in the Bcl-2 protein family and associates with drug resistance in human lymphomas, while the Bax, Bad and Bid proteins are pro-apoptotic members [32, 33].

We examined whether Mfn2-mediated activation of caspase-3 was affected by the cellular level of the Bcl-2 family proteins. The cervical tumour tissues injected with AdvMfn2 or Adv-control were prepared for testing. The results represented in Fig. 5D and 5E demonstrated that the overexpression of Mfn2 could not only increase the levels of Bax and Bid in cervical tumour tissues but also decrease the phosphorylation of Bad and the expression of Bcl-2.

Therefore, we concluded that Mfn 2 apparently induced apoptosis by activating caspase- 3 through the mitochondrial apoptosis pathway. Nevertheless, the fact that the mitochondrial outer-membrane protein Mfn-2 highly increased in cervical tumour cells, demonstrated that apoptosis occurred via the mitochondrial apoptosis pathway.

\section{Discussion}

In the current study, we confirmed the cell apoptotic effect of Mfn2 on cervical carcinoma HeLa cells in vitro and in vivo. Data from CCK-8 and flow cytometry showed that Mfn 2 could inhibit proliferation and induce apoptosis in a dose- and time-dependent manner in HeLa cells and C33A cells. The JC- 1 Kit assay results revealed that the membrane potential of the mitochondria decreased in a dose-dependent manner in HeLa cells after Adv-Mfn2 treatment. The Western blot data confirmed higher cytosolic amounts of cytochrome c with increasing doses of Adv-Mfn2, which signified the onset of the intrinsic apoptotic pathway. To further validate apoptotic control by Mfn2, expression levels of important apoptotic proteins were analysed by Western blot. Levels of cleaved caspase- 3 and cleaved caspase- 9 increased in HeLa cells with Adv-Mfn2 treatment. We also found significant increases in Bax levels and decreased Bcl-2 levels with Adv-Mfn2 treatment. These are consistent with findings from the data, which confirmed that Mfn2 could induce apoptosis in the mitochondrial pathway in vivo. We examined the cervical tumour tissues from the nude mouse model of HeLa cells in the Ad-mfn2 group, of which p53 expression is upregulated. In HeLa cells, Mfn2 may inhibit HPV E6, which is combined with p53 [34], and inhibits the proliferation of HeLa cells. Meanwhile, Mfn2 is a novel direct target of p53 [35], and there may be positive feedback between Mfn2 with p53 [36]. Mfn2 may inhibit C33A cell proliferation through a positive feedback relationship with Mfn2 and p53. We also found that Mfn2 could inhibit cervical tumour size after injection with Ad-mfn2 and induce cervical tumour cells into apoptosis. To discuss the molecular mechanism of Mfn2 in triggering cervical tumour apoptosis, we analysed the expression of $\mathrm{Bcl}-2$ family proteins. The results showed that $\mathrm{Mfn} 2$ upregulated pro-apoptotic proteins of Bcl-2 family members as Bax in the mitochondrial apoptosis pathway and downregulated anti-apoptotic proteins such as Bcl-2. These preclinical studies all revealed the therapeutic potential of the Mfn2 protein for anti-cancer treatment.

Mitochondria, the intra-cellular unique DNA-containing organelle whose most common function is the production of adenosine triphosphate (ATP) in the presence of oxygen, is also a biologically important source and target for reactive species. Thus, mitochondria play a key role in cancer and its progression. Except for regulating mitochondrial fusion, the mitochondrial outer membrane protein Mfn2 has been proved pivotal in maintaining mitochondrial morphology and homeostasis. In addition, studies have shown that Mfn2 regulates cell proliferation and apoptosis associated with mitochondria [13]. The cytosolic release of cytochrome $c$, which inflicts an irrevocable activation of caspase and subsequent cell death, which is due to the damaged mitochondrial membrane $[37,38]$. Although the 
mechanisms regulating mitochondrial permeability and the release of cytochrome c during apoptosis are not fully understood, Bcl-xL, Bcl-2, and Bax may influence the voltagedependent anion channels, which may play a role in regulating cytochrome $\mathrm{c}$ release. The pro-apoptotic Bcl-2 proteins Bad, Bid, Bax, and Bim may reside in the cytosol but translocate to mitochondria following death signalling, where they promote the release of cytochrome $c$. Bad translocates to mitochondria and forms a pro-apoptotic complex with Bcl-xL.

$\mathrm{Bcl}-2$ is a major anti-apoptotic Bcl-2 family member that is directly involved in the suppression of apoptosis at the decision stage [39]. Bcl-2can inhibit apoptosis induced by p53 [40]. On the other hand, Bax is a major pro-apoptotic Bcl-2 family member that has been identified as a promising prognostic indicator in patients with lung cancer [41], and a molecule required for cell death that is extensively expressed in both small cell (SCLC) and non-small cell lung cancer (NSCLC) cells [42-44], suggesting that it could potentially also offer a therapeutic target in cancer patients. The target, which influences the expression and function of Bax and Bcl-2, could affect cancer and its progression. Mimicking the BH3 domain to induce apoptosis has recently been used as a strategy for the development of Bcl2 inhibitors as anticancer drugs [45-48]. The BH3 mimetic agents function as competitive inhibitors by binding to the hydrophobic cleft of Bcl-2/Bcl-XL [45, 48]. Four Bcl-2 inhibitors have already been tested in human clinical trials but showed limited clinical efficacy [42, 43, $45,46]$. The multi-domain pro-apoptotic Bax provides the required gateway to apoptotic cell death $[48,49]$. Many times, it was focused on either Bax upregulation or Bcl-2 inhibition. In mitochondria, Mfn2 upregulated pro-apoptotic Bax and downregulated anti-apoptotic Bcl-2 at the same time, inducing HeLa cells into apoptosis via the mitochondrial pathway.

We found that Mfn2 could activate Bax and inhibit Bcl-2 expression in the mitochondrial pathway, which provided a strong rationale that Mfn2 combined anti-apoptotic protein inhibition and pro-apoptotic protein activation, which should have superior therapeutic benefits, and effectively triggered tumour cells into apoptosis. Based on our data, we propose that Mfn2, which co-targets pro-apoptotic and anti-apoptotic proteins in the mitochondrial pathway, offers a more effective strategy for cervical carcinoma treatment.

\section{Conclusion}

Our studies demonstrated that Mfn2 can induce cervical carcinoma HeLa cell apoptosis via the mitochondrial pathway. It activated the pro-apoptotic processes in the mitochondrial apoptosis pathway and inhibited anti-apoptotic protein expression in the mitochondrial pathway. All these features illustrate that Mfn2 may be a promising starting point for the future clinical application of efficient anticancer treatment targets in the cervical carcinoma therapy.

\section{Acknowledgements}

Our animal studies were carried out according to the Guide for the Care and Use of Laboratory Animals of the People's Republic of China in strict compliance. All efforts were made to minimize suffering and all procedures were performed under ethyl ether anaesthesia. The study protocol was approved by the Committee on the Ethics of Animal Experiments of the Chinese Centre for Diseases Control and Prevention.

We thank Chun Liu, Jingwen Yu, Jun Sun, Chao Luo and Jun Yang for helpful discussion.

\section{Disclosure Statement}

This study was sponsored by the National Natural Science Foundation of China for Youth (No. 81402568; No. 81401429) to X.W.L.

\section{KARGER}




\section{Cellular Physiology Cell Physiol Biochem 2018;46:69-81

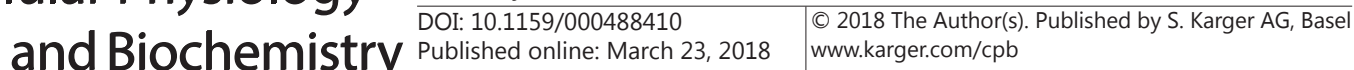

Wang et al.: Mfn2 Induces Hela Cells into Apoptosis

\section{References}

1 Torre LA, Bray F, Siegel RL, Ferlay J, Lortet-Tieulent J, Jemal A: Global cancer statistics, 2012. CA Cancer J Clin 2015;65:87-108.

2 Zugazagoitia J, Guedes C, Ponce S, Ferrer I, Molina-Pinelo S, Paz-Ares L: Current challenges in cancer treatment. Clin Ther 2016;38:1551-1566.

3 Hanahan D, Weinberg RA: The hallmarks of cancer. Cell 2000;100:57-70.

4 Hanahan D, Weinberg RA: Hallmarks of cancer: The next generation. Cell 2011;144:646-674.

5 Adams JM, Cory S: The bcl-2 apoptotic switch in cancer development and therapy. Oncogene 2007;26:13241337.

-6 Chen H, Detmer SA, Ewald AJ, Griffin EE, Fraser SE, Chan DC: Mitofusins mfn1 and mfn2 coordinately regulate mitochondrial fusion and are essential for embryonic development. J Cell Biol 2003;160:189-200.

7 de Brito OM, Scorrano L: Mitofusin 2: A mitochondria-shaping protein with signaling roles beyond fusion. Antioxid Redox Signal 2008;10:621-634.

8 Bereiter-Hahn J, Vöth M: Dynamics of mitochondria in living cells: Shape changes, dislocations, fusion, and fission of mitochondria. Microsc Res Tech 1994;27:198-219.

-9 Youle RJ, van der Bliek AM: Mitochondrial fission, fusion, and stress. Science 2012;337:1062-1065.

10 Campello S, Scorrano L: Mitochondrial shape changes: Orchestrating cell pathophysiology. EMBO Rep 2010;11:678-684.

11 Santel A, Fuller MT: Control of mitochondrial morphology by a human mitofusin. J Cell Sci 2001;114:867874.

12 Santel A: Get the balance right: Mitofusins roles in health and disease. Biochim Biophys Acta 2006;1763:490-499.

13 Guo X, Chen KH, Guo Y, Liao H, Tang J, Xiao RP: Mitofusin 2 triggers vascular smooth muscle cell apoptosis via mitochondrial death pathway. Circ Res 2007;101:1113-1122.

14 Wang W, Xie Q, Zhou X, Yao J, Zhu X, Huang P, Zhang L, Wei J, Xie H, Zhou L, Zheng S: Mitofusin-2 triggers mitochondria ca2+ influx from the endoplasmic reticulum to induce apoptosis in hepatocellular carcinoma cells. Cancer Lett 2015;358:47-58.

15 Zhou X, Zhang L, Zheng B, Yan Y, Zhang Y, Xie H, Zhou L, Zheng S, Wang W: Microrna-761 is upregulated in hepatocellular carcinoma and regulates tumorigenesis by targeting mitofusin-2. Cancer Sci 2016;107:424432.

16 Chen K-H, Guo X, Ma D, Guo Y, Li Q, Yang D, Li P, Qiu X, Wen S, Xiao R-P, Tang J: Dysregulation of hsg triggers vascular proliferative disorders. Nat Cell Biol 2004;6:872-883.

17 Kannan A, Wells RB, Sivakumar S, Komatsu S, Singh KP, Samten B, Philley JV, Sauter ER, Ikebe M, Idell S, Gupta S, Dasgupta S: Mitochondrial reprogramming regulates breast cancer progression. Clin Cancer Res 2016;22:3348-3360.

18 Züchner S, Mersiyanova IV, Muglia M, Bissar-Tadmouri N, Rochelle J, Dadali EL, Zappia M, Nelis E, Patitucci A, Senderek J, Parman Y, Evgrafov O, Jonghe PD, Takahashi Y, Tsuji S, Pericak-Vance MA, Quattrone A, Battologlu E, Polyakov AV, Timmerman V, Schröder JM, Vance JM: Mutations in the mitochondrial gtpase mitofusin 2 cause charcot-marie-tooth neuropathy type 2a. Nat Genet 2004;36:449-451.

19 Karbowski M, Lee YJ, Gaume B, Jeong SY, Frank S, Nechushtan A, Santel A, Fuller M, Smith CL, Youle RJ: Spatial and temporal association of bax with mitochondrial fission sites, drp1, and $\mathrm{mfn} 2$ during apoptosis. J Cell Biol 2002;159:931-938.

20 Cheung TH, Lo KW, Yim SF, Poon CS, Cheung AY, Chung TK, Wong YF: Clinicopathologic significance of loss of heterozygosity on chromosome 1 in cervical cancer. Gynecol Oncol 2005;96:510-515.

-21 Bagchi A, Mills AA: The quest for the 1p36 tumor suppressor. Cancer Res 2008;68:2551-2556.

22 Jin B, Fu G, Pan H, Cheng X, Zhou L, Lv J, Chen G, Zheng S: Anti-tumour efficacy of mitofusin-2 in urinary bladder carcinoma. Med Oncol 2011;28:S373-380.

23 Wu L, Li Z, Zhang Y, Zhang P, Zhu X, Huang J, Ma T, Lu T, Song Q, Li Q, Guo Y, Tang J, Ma D, Chen KH, Qiu X: Adenovirus-expressed human hyperplasia suppressor gene induces apoptosis in cancer cells. Mol Cancer Ther 2008;7:222-232.

24 Wang W, Lu J, Zhu F, Wei J, Jia C, Zhang Y, Zhou L, Xie H, Zheng S: Pro-apoptotic and anti-proliferative effects of mitofusin-2 via bax signaling in hepatocellular carcinoma cells. Med Oncol 2012;29:70-76.

25 Dasgupta A, Chen K-H, Munk RB, Sasaki CY, Curtis J, Longo DL, Ghosh P: Mechanism of activation-induced 


\section{Cellular Physiology Cell Physiol Biochem 2018;46:69-81

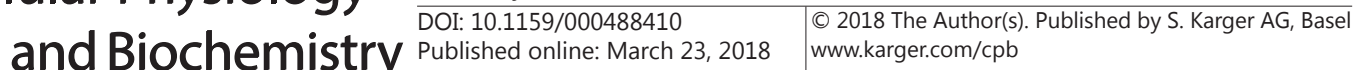

Wang et al.: Mfn2 Induces Hela Cells into Apoptosis

downregulation of mitofusin 2 in human peripheral blood t cells. J Immunol 2015;195:5780-5786.

26 Liu X-W, Yuan P, Tian J, Li L-J, Wang Y, Huang S-C, Liu L, Backendorf C, Noteborn MHM, Sun J: Ptd4-apoptin induces bcl-2-insensitive apoptosis in human cervical carcinoma in vitro and in vivo. Anticancer Drugs 2016;27:979-987.

27 Ye H, Liu X, Lv M, Wu Y, Kuang S, Gong J, Yuan P, Zhong Z, Li Q, Jia H, Sun J, Chen Z, Guo A-Y: Microrna and transcription factor co-regulatory network analysis reveals mir-19 inhibits cyld in t-cell acute lymphoblastic leukemia. Nucleic Acids Res 2012;40:5201-5214.

-28 Sun J, Yan Y, Wang X-T, Liu X-W, Peng D-J, Wang M, Tian J, Zong Y-Q Zhang Y-H, Noteborn MHM, Qu S: Ptd4apoptin protein therapy inhibits tumor growthin vivo. Int J Cancer 2009;124:2973-2981.

-29 Frenzel A, Grespi F, Chmelewskij W, Villunger A: Bcl2 family proteins in carcinogenesis and the treatment of cancer. Apoptosis 2009;14:584-596.

30 Kroemer G, Susin SA, Lorenzo HK, Zamzami N, Marzo I, Snow BE, Brothers GM, Mangion J, Jacotot E, Costantini P, Loeffler M, Larochette N, Goodlett DR, Aebersold R, Siderovski DP, Penninger JM: Molecular characterization of mitochondrial apoptosis-inducing factor. Nature 1999;397:441-446.

31 Qiu JH, Asai A, Chi S, Saito N, Hamada H, Kirino T: Proteasome inhibitors induce cytochrome c-caspase-3like protease-mediated apoptosis in cultured cortical neurons. J Neurosci 2000;20:259-265.

32 Krishna S, Low ICC, Pervaiz S: Regulation of mitochondrial metabolism: Yet another facet in the biology of the oncoprotein bcl-2. Biochem J 2011;435:545-551.

33 Ekoff M, Nilsson G: Mast cell apoptosis and survival. Adv Exp Med Biol 2011;716:47-60.

34 Pang CL, Thierry F: Human papillomavirus proteins as prospective therapeutic targets. Microb Pathog 2013;58:55-65.

35 Wang W, Cheng X, Lu J, Wei J, Fu G, Zhu F, Jia C, Zhou L, Xie H, Zheng S: Mitofusin-2 is a novel direct target of p53 Biochem Biophys Res Commun 2010;400:587-592.

-36 Wu Y, Zhou D, Xu X, Zhao X, Huang P, Zhou X, Song W, Guo H, Wang W, Zheng S: Clinical significance of mitofusin-2 and its signaling pathways in hepatocellular carcinoma. World J Surg Oncol 2016;14:179.

-37 Cadenas E: Mitochondrial free radical production and cell signaling. Mol Aspects Med 2004;25:17-26.

-38 Simon HU, Haj-Yehia A, Levi-Schaffer F: Role of reactive oxygen species (ros) in apoptosis induction. Apoptosis 2000;5:415 - 418.

-39 Deng X, Gao F, Flagg T, Anderson J, May WS: Bcl2's flexible loop domain regulates p53 binding and survival. Mol Cell Biol 2006;26:4421-4434.

40 Estaquier J, Vallette F, Vayssiere JL, Mignotte B: The mitochondrial pathways of apoptosis. Adv Exp Med Biol 2012;942:157-183.

41 Groeger AM, Esposito V, Cassandro R, Baldi G, Rossiello L, De Luca L, Kadletz M, Kaiser HE: A model of bax gene delivery to human lung cancer. Anticancer Res 2001;21:3627-3630.

42 Wei MC: Proapoptotic bax and bak: A requisite gateway to mitochondrial dysfunction and death. Science 2001;292:727-730.

43 Xin M, Deng X: Nicotine inactivation of the proapoptotic function of bax through phosphorylation. J Biol Chem 2005;280:10781-10789.

44 Xin M, Deng X: Protein phosphatase 2a enhances the proapoptotic function of bax through dephosphorylation. J Biol Chem 2006;281:18859-18867.

45 Chonghaile TN, Letai A: Mimicking the bh3 domain to kill cancer cells. Oncogene 2008;27:S149-S157.

46 Kang MH, Reynolds CP: Bcl-2 inhibitors: Targeting mitochondrial apoptotic pathways in cancer therapy. Clin Cancer Res 2009;15:1126-1132.

-47 Oltersdorf T, Elmore SW, Shoemaker AR, Armstrong RC, Augeri DJ, Belli BA, Bruncko M, Deckwerth TL, Dinges J, Hajduk PJ, Joseph MK, Kitada S, Korsmeyer SJ, Kunzer AR, Letai A, Li C, Mitten MJ, Nettesheim DG, Ng S, Nimmer PM, O'Connor JM, Oleksijew A, Petros AM, Reed JC, Shen W, Tahir SK, Thompson CB, Tomaselli KJ, Wang B, Wendt MD, Zhang H, Fesik SW, Rosenberg SH: An inhibitor of bcl-2 family proteins induces regression of solid tumours. Nature 2005;435:677-681.

48 Xin M, Li R, Xie M, Park D, Owonikoko TK, Sica GL, Corsino PE, Zhou J, Ding C, White MA, Magis AT, Ramalingam SS, Curran WJ, Khuri FR, Deng X: Small-molecule bax agonists for cancer therapy. Nat Commun 2014;5:4935.

49 Han B, Park D, Li R, Xie M, Owonikoko TK, Zhang G, Sica GL, Ding C, Zhou J, Magis AT, Chen ZG, Shin DM, Ramalingam SS, Khuri FR, Curran WJ, Deng X: Small-molecule bcl2 bh4 antagonist for lung cancer therapy. Cancer Cell 2015;27:852-863. 